\title{
Primary Culture of Choroid Plexuses from Neonate Rats Containing Progenitor Cells Capable of Differentiation
}

\author{
Sheng-Li Huang ${ }^{1}$, Xi-Jing He ${ }^{1}$, Zong-Fang Li ${ }^{2}$, Lu Yao ${ }^{3}$, Guo-Lian Yuan², Wei Shi ${ }^{4}$ \\ ${ }^{1}$ Department of Orthopaedics, the Second Affiliated Hospital, Xi'an Jiaotong University School of Medicine, Xi'an, China \\ ${ }^{2}$ Central Laboratory for Scientific Research, the Second Affiliated Hospital, Xi'an Jiaotong University School of Medicine, Xi'an, China \\ ${ }^{3}$ Institute of Neurobiology, Xi'an Jiaotong University School of Medicine, Xi'an, China \\ ${ }^{4}$ Department of Neurosurgery, the Second Affiliated Hospital, Xi'an Jiaotong University School of Medicine, Xi'an, China
}

\begin{abstract}
Background: The choroid plexuses, which could secrete a number of neurotrophins, have recently been used in transplantation in central nervous system diseases.

Aims: To study the mechanism of nerve regeneration in the central nervous system by grafting choroid plexus tissues.

Study Design: Animal experimentation.

Methods: The choroid plexuses from the lateral ventricles of neonatal rats were cultured in adherent culture, and immunocytochemical methods were used to analyse the progenitor cells on days 2, 6, and 10 after seeding.

Results: Expression of both nestin and glial fibrillary acidic protein was observed in small cell aggregates on day 2 in primary culture. Most of the nestinpositive cells on day 6 were immunoreactive to glial fibrillary acidic protein antibody. No cells expressing nestin or glial fibrillary acidic protein were seen on day 10.

Conclusion: These experimental results indicate that the choroid plexus contains a specific cell population - progenitor cells. Under in vitro experimental conditions, the progenitor cells differentiated into choroid plexus epithelial cells but did not form neurons or astrocytes.
\end{abstract}

Key Words: Choroid plexus, primary culture, progenitor cells, differentiation, rat

Received: 27.11 .2012

Accepted: 13.06 .2013

\section{Introduction}

The choroid plexuses within the brain ventricles, which are small yet complex organs, are known to produce cerebrospinal fluid and secrete an expanding number of growth factors, neurotrophins, and other neuroprotective compounds (1-3). The choroid plexus consists of an outer epithelial layer and an inner core of stromal cells. The choroid plexus epithelial cells possess secretary capability that makes them attractive candidates for transplantation to minimise or prevent neural degeneration and its functional consequences.

Presently, primary culture from choroid plexus tissues is widely used in transplantation in animal models for central nervous system diseases (4-11). The cellular and molecular mechanisms of nerve regeneration in the central nervous system after choroid plexus transplantation have not been defined. Fortunately, primary culture offers the opportunity for visualising many processes, such as nerve repair, at the level of single cells to better understand the cell biology. Immunocytochemical methods are frequently used for this purpose.

The feasibility of cultivation of dissociated choroid plexus epithelial cells prepared from neonate rats has been demonstrated in our previous work (12). Neural stem cells have been identified in choroid plexuses of neonate rats, and large num- bers of nestin-expressing cells persist in the choroid plexuses (13). In this study, we assessed the presence of nestin-positive cells in primary cultures from choroid plexuses of neonate rats in order to investigate whether progenitor cells were present in the culture.

\section{Material and Methods}

\section{Animals}

Neonatal Sprague-Dawley rats of both sexes (postnatal day 1) were supplied by the Center of Experimental Animals, $X i$ an Jiaotong University School of Medicine. The protocols for animal care and experimental management were approved by the Animal Experimentation Committee of Xi'an Jiaotong University. Ethical approval for the study was obtained from the Ethics Committee of the Second Affiliated Hospital, School of Medicine, Xi'an Jiaotong University. Principles of laboratory animal care were followed and all procedures were conducted according to the guidelines established by the $\mathrm{Na}$ tional Institutes of Health.

\section{Reagents}

Dulbecco's modified Eagle's medium (DMEM, low glucose) and foetal bovine serum (FBS) were purchased from 

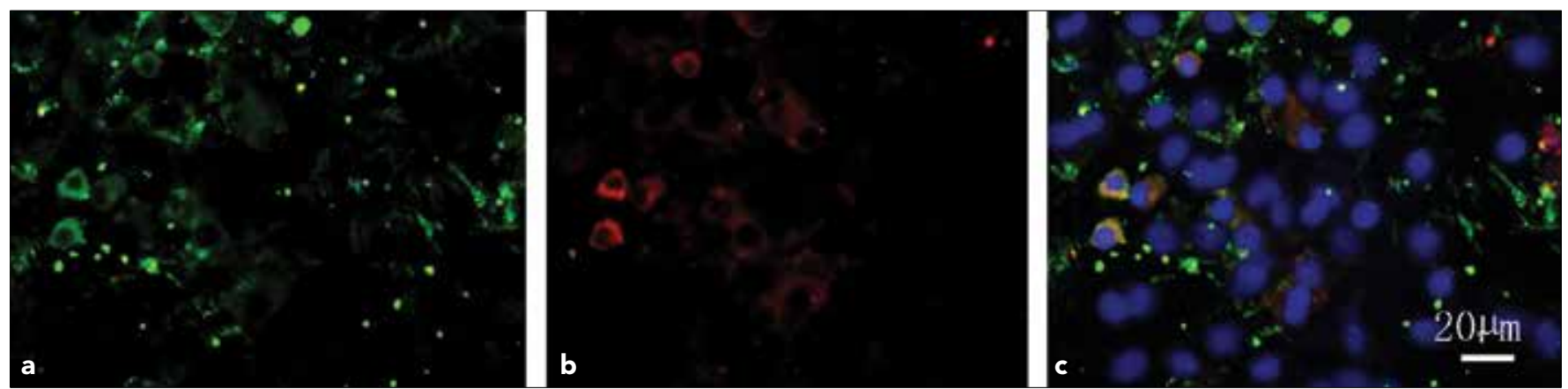

Figure 1. a-c. Primary culture of choroid plexuses on day 2 after seeding of dissociated cells $(\times 400)$. Nestin staining in green (a). GFAP staining in red (b). Nuclear labelling in blue and merged images (c)

Thermo, and 24-well plates were provided by Corning Costar. Rabbit anti-transthyretin (TTR) was purchased from Santa Cruz and 4',6-diamino-2-phenylindole (DAPI) was purchased from Roche. Poly-L-lysine and rabbit anti-glial fibrillary acidic protein (GFAP) were purchased from Sigma-Aldrich. Recombinant rat epidermal growth factor (EGF) was obtained from PeproTech. Mouse anti-nestin monoclonal antibody, fluorescein-conjugated goat anti-mouse immunoglobulin $\mathrm{G}(\mathrm{lg} \mathrm{G})$, and Cy3-conjugated goat anti-rabbit IgG were obtained from Millipore.

\section{Cell culture}

Primary choroid plexus epithelial cells were cultured following the procedure we described earlier (12). Briefly, each neonate rat was sacrificed, the brain was removed from the skull, and choroid plexuses were removed from the lateral ventricle and minced mechanically. The obtained suspension was pelleted by centrifugation at $800 \mathrm{rpm}$ for $5 \mathrm{~min}$, and the pellets were resuspended in culture media consisting of DMEM supplemented with $10 \%$ (v/v) FBS, $10 \mathrm{ng} / \mathrm{mL}$ EGF, and antibiotics (penicillin/streptomycin). The cells were then seeded in 24-well plates with glass coverslips coated in advance with poly-L-lysine following the manufacturer's suggestions. The dissociated cells were then incubated in a $5 \%$ humidified carbon dioxide incubator at $37^{\circ} \mathrm{C}$. After $48 \mathrm{~h}$, the medium was removed and fresh medium was added to the culture. Thereafter, the culture medium was replaced every 3 days. All cultured cells were examined daily for growth under a phase contrast microscope.

\section{Immunocytology}

The cells were fixed and evaluated on days 2, 6, and 10 after seeding, and adherent cells were immunolabelled for markers including nestin, GFAP, and TTR to identify the cell types. After fixation in $4 \%$ paraformaldehyde for 10 $\mathrm{min}$ at room temperature, the coverslips were washed with phosphate-buffered saline three times for 5 min each time. Nonspecific reactions were blocked with $10 \%$ normal goat serum for $30 \mathrm{~min}$. The cells were subsequently incubated at $4^{\circ} \mathrm{C}$ overnight with the following primary antibodies: mouse anti-nestin monoclonal antibody (1:200) and GFAP (1:200). After three washes in phosphate-buffered saline, secondary antibodies, fluorescein-conjugated goat anti-mouse lgG, and Cy3-conjugated goat anti-rabbit IgG were added, and the
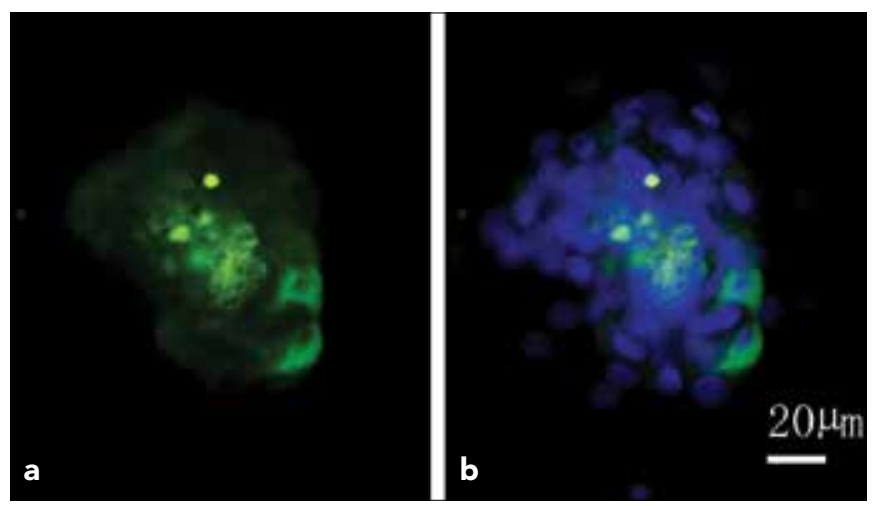

Figure 2. a, b. Primary culture of choroid plexuses on day 2. Nestin-positive cells are often found located adjacent to choroid plexus epithelial cells $(\times 400)$. Nestin staining in green (a). Nuclear labelling in blue and merged images (b)

cell-seeded coverslips were incubated for $2 \mathrm{~h}$ at room temperature. Cell nuclei were counterstained using DAPI. Then the sections were washed in phosphate-buffered saline again, air-dried, and coverslipped. Fluorescence microscopy was carried out by using an Olympus BX51 microscope equipped with a mercury lamp power supply. A negative control was set up by omitting primary antibodies. The cultured sections on day 10 for immunocytochemical staining were also used for detection of nestin and TTR following the method described above.

\section{Results}

The expression of nestin was used as the primary marker of progenitor-like cells in our studies and the immunoreactivity of GFAP, another marker, was evaluated. TTR immunolabelling was also investigated.

Under a phase contrast microscope, we observed that the cultured cells migrated from tissue clusters and formed large colonies of choroid plexus epithelial cells. The expression of both nestin and GFAP was observed in the small cell aggregates from the primary cultures of choroid plexuses as early as 2 days after seeding (Figure 1). Nestin-expressing cells were found adjacent to the choroid plexus epithelial cells or inside the cell aggregates (Figure 2). When the cells were continuously cultured, nestin-positive cells continued proliferating and migrated gradually into the surrounding areas. Interest- 


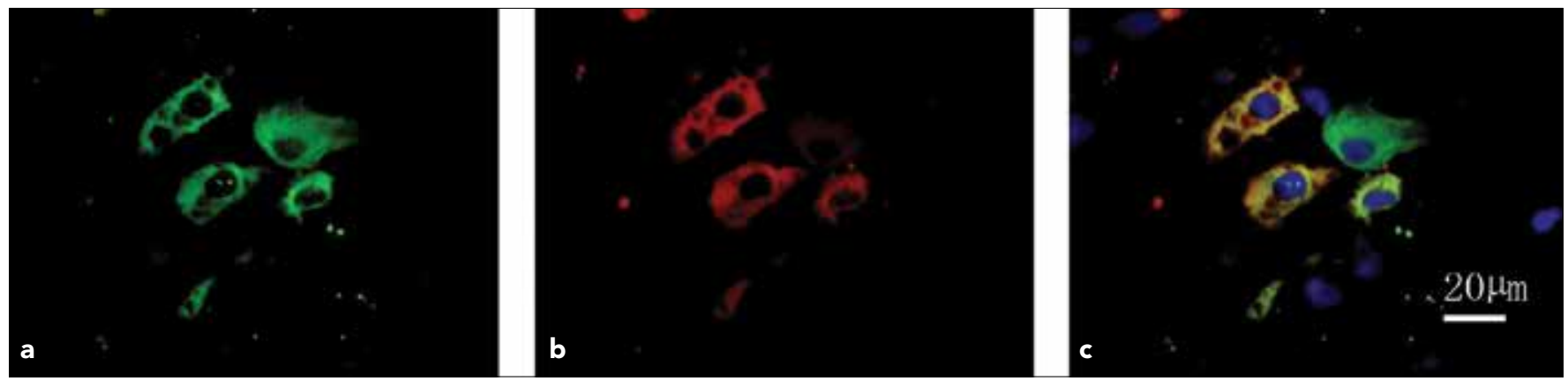

Figure 3. a-c. Six-day-old primary culture of choroid plexuses. Most nestin-positive cells are co-labelled with GFAP $(\times 400)$. Nestin staining in green (a). GFAP staining in red (b). Nuclear labelling in blue and merged images (c)

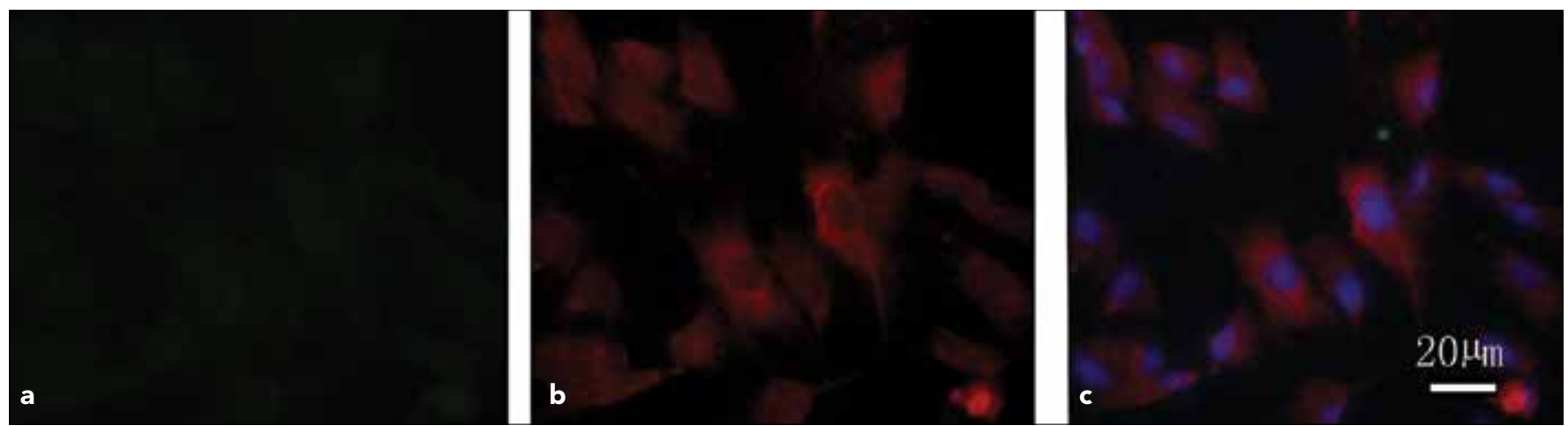

Figure 4. a-c. Primary cultures of choroid plexuses on day 10. Cells are positive for TTR and negative for nestin ( $x 400)$. Nestin staining in green (a). TTR staining in red (b). Nuclear labelling in blue and merged images (c)

ingly, nestin-positive cells could be co-labelled with GFAP. We found that most of the nestin-positive cells in the cultures on day 6 were immunoreactive to the GFAP antibody (Figure 3). After 6 days of cultivation, some of the nestin-positive cells did not co-express GFAP, indicating an abrupt down-regulation of GFAP expression when progenitor cells started to differentiate into choroid plexus epithelial cells. From day 10, the cells were not immunostained with either nestin or GFAP. The choroid plexus epithelial cells were ascertained by staining with an anti-TTR antibody. Under our experimental conditions, almost $100 \%$ of the cells from day 10 were positive for TTR (Figure 4).

\section{Discussion}

Primary tissue culture has played an important role in revealing the properties and potential of choroid plexus epithelial cells in the normal brain. The present paper, to our knowledge, is the first to demonstrate progenitor cells derived from primary culture of neonate choroid plexuses. We show here that this cell population has a high proliferative potential, that is to say, the primary cultures from choroid plexuses contain progenitor cells that differentiate into choroid plexus epithelial cells in vitro.

The choroid plexus acts as a site of neurogenesis (11, 1419). Transplantation of the choroid plexus has been tested in animal models in recent years. In all the reported studies, the grafts of choroid plexus cells from primary culture were shortterm (approximately 2 days). Therefore, our results based on 10-day cultivation will be helpful in understanding the mechanism of transplantation of choroid plexuses cultured for a short time. It was found in our previous study that the choroid plexus epithelial cells from primary culture became confluent on day 10 (12); as a result, we set 10 days as the length of culture in this study to obtain a sufficient number of choroidal epithelial cells for transplantation. In the published studies, cell clusters (primarily composed of epithelial cells) that included portions of the undigested fibroblastic stroma rather than grafts of purified epithelial cells were used. In general, the mechanism by which choroid plexus transplants exert their beneficial effect is through secreting proteins with trophic activity. In choroid plexus cultures obtained from the lateral ventricles, nestin was detectable during the period of proliferation and maturation. This indicates that the choroid plexus contains a specific cell population - progenitor cells. Our study reveals a mechanism of transplantation of primary cultures from choroid plexuses, namely, progenitor cells existing in the primary cultures, and further suggests a promising prospect of choroid plexus transplantation treatment in central nervous system diseases.

Nestin is a type VI intermediate filament protein originally described in neural stem cells (20) and is therefore taken as a marker for neural stem cells. Though nestin-positive cells are difficult to identify by phase contrast microscopy, immunocytochemistry can reveal the existence of these cells in choroid plexus primary culture. During cell differentiation, nestin expression is transient, and it is down-regulated after differentiation. The reason for this might be that the proportion of 
cells expressing different antigenic markers is also affected by culture conditions. In this study, almost $100 \%$ of the cells in the cultures on day 10 were positive for TTR and the choroid plexus epithelial cells did not exhibit immunoreactivity for nestin or GFAP. This indicates that only choroid plexus epithelial cells and not neurons and astrocytes can be generated. DMEM, to a lesser extent, also induces the proliferation of precursor cells in cultures of choroid plexuses derived from the ventricle of neonate animals. Subject to the local microenvironment and developmental stages, such precursors may differentiate into choroid plexus epithelial cells.

Immunocytochemical study of the expression of nestin and GFAP at different time points showed the process of nestin-positive cells differentiating into choroid plexus epithelial cells. The complex processes in the cells that developed over a period of 2-10 days of culturing were revealed. The primary culture of choroid plexus epithelial cells should be considered as a mixture of cells arising from dissociated cells. We have found in the present experiments that choroid plexuses in neonate rats contain large numbers of precursor cells, and that the neonate cultures contain nestin-positive cells. Grafts of choroid plexus cells from neonatal and young animals appear to be beneficial (21). Therefore, neonatal animals are the optimal source of primary culture.

Since choroid plexuses can be distinguished by their specific location and are related to ventricular cavities, contamination of the cerebral parenchymal tissues can be avoided in the dissection of the choroid plexuses from the lateral ventricles of rats. In addition, to exclude the possibility that non-cell structures from the choroid plexus contributed to the production of nestin-positive cells, we used an antibody (anti-TTR) specific to choroid plexus epithelial cells as a marker to detect cell purity.

It is important to note that despite the similarities in the strong nestin immunoreactivity, there are fundamental differences between sphere and choroid plexus epithelial cells in the primary culture. A complete understanding of the physiological role of these cells is still lacking. We have observed in vivo that during early postnatal development, progenitor cells are specifically located within the choroid plexus (13). Therefore, it is conceivable that nestin-positive cells in the choroid plexuses form a subpopulation of specialised cells, and choroid plexus stroma may provide an adequate environment for the survival, proliferation, and differentiation of progenitor cells.

In conclusion, we report here for the first time to our knowledge that primary culture of choroid plexus epithelial cells has nestin-expressing cells. The choroid plexus contains a specific pool of cells that behave in vitro as precursor cells of choroid plexus epithelial cells. It is difficult to escape the conclusion that nestin-positive cells arising from neonate rat choroid plexuses can develop into choroid plexus epithelial cells in vitro. Thus, an increased understanding of the cells involved in the choroid plexuses will help identify new mechanisms for developing potential therapeutic approaches to reducing functional deficits and improving recovery in central nervous system diseases. Meanwhile, the primary culture system for the choroid plexus is useful for investigation of cell differentiation under in vitro conditions.
Ethics Committee Approval: Ethics committee approval was received for this study from the Ethics Committee of the Second Affiliated Hospital, School of Medicine, Xi'an Jiaotong University.

\section{Informed Consent: N/A}

Peer-review: Externally peer-reviewed.

Author contributions: Concept-S.L.H., W.S.; Design - S.L.H., X.J.H., W.S.; Supervision - W.S.; Resource - S.L.H., W.S.; Materials - S.L.H., X.J.H., Z.F.L., L.Y., G.L.Y.; Data Collection\&/or Processing - S.L.H., L.Y., G.L.Y.; Analysis\&/or Interpretation - S.L.H., X.J.H., Z.F.L., L.Y., G.L.Y., W.S.; Literature Search - S.L.H., Z.F.L., L.Y.; Writing - S.L.H.; Critical Reviews - X.J.H., Z.F.L., L.Y., G.L.Y., W.S.

Conflict of Interest: No conflict of interest was declared by the authors.

Financial Disclosure: This study was supported by research grants from the National Natural Science Foundation of China (No. 81271340 and No.30973099)

\section{References}

1. Chodobski A, Szmydynger-Chodobska J. Choroid plexus: target for polypeptides and site of their synthesis. Microsc Res Tech 2001;52:65-82. [CrossRef]

2. Emerich DF, Vasconcellos AV, Elliott RB, Skinner SJM, Borlongan CV. The choroid plexus:function, pathology and therapeutic potential of its transplantation. Expert Opin Biol Ther 2004;4:1191-201. [CrossRef]

3. Emerich DF, Skinner SJM, Borlongan CV, Vasconcellos AV, Thanos CG. The choroid plexus in the rise, fall, and repair of the brain. Bioessays 2005;27:262-74. [CrossRef]

4. Ide C, Kitada M, Chakrabortty S, Taketomi M, Matsumoto N, Kikukawa $\mathrm{S}$, et al. Grafting of choroid plexus ependymal cells promotes the growth of regenerating axons in the dorsal funiculus of rat spinal cord:a preliminary report. Exp Neurol 2001;167:242-51. [CrossRef]

5. Borlongan CV, Thanos CG, Skinner SJ, Geaney M, Emerich DF. Transplants of encapsulated rat choroid plexus cells exert neuroprotection in a rodent model of Huntington's disease. Cell Transplant 2008;16:987-92. [CrossRef]

6. Emerich DF, Thanos CG, Goddard M, Skinner SJ, Geany MS, Bell WJ, et al. Extensive neuroprotection by choroid plexus transplants in excitotoxin lesioned monkeys. Neurobiol Dis 2006;23:471-80. [CrossRef]

7. Emerich DF, Thanos CG. In vitro culture duration does not impact the ability of encapsulated choroid plexus transplants to prevent neurological deficits in an excitotoxin-lesioned rat model of Huntington's disease. Cell Transplant 2006;15:595-602. [CrossRef]

8. Borlongan CV, Skinner SJ, Geaney M, Vasconcellos AV, Elliott RB, Emerich DF. Neuroprotection by encapsulated choroid plexus in a rodent model of Huntington's disease. Neuroreport 2004;15:2521-5. [CrossRef]

9. Borlongan CV, Skinner SJ, Geaney M, Vasconcellos AV, Elliott RB, Emerich DF. CNS grafts of rat choroid plexus protect against cerebral ischemia in adult rats. Neuroreport 2004;15:1543-7. [CrossRef]

10. Borlongan CV, Skinner SJ, Geaney M, Vasconcellos AV, Elliott $\mathrm{RB}$, Emerich DF. Intracerebral transplantation of porcine choroid plexus provides structural and functional neuroprotection in a rodent model of stroke. Stroke 2004;35:2206-10. [CrossRef]

11. Kitada M, Chakrabortty S, Matsumoto N, Taketomi M, Ide C. Differentiation of choroid plexus ependymal cells into astrocytes after grafting into the pre-lesioned spinal cord in mice. Glia 2001;36:364-74. [CrossRef]

12. Huang SL, He XJ, Li ZF, Yao L, Shi W. A novel primary culture method for rat choroidal epithelial cells. Neurosciences (Riyadh) 2013;18:27-32. 
13. Huang SL, Shi W, Jiao $Q$, He XJ. Change of neural stem cells in the choroid plexuses of developing rat. Int $\mathrm{J}$ Neurosci 2011;121:350-5. [CrossRef]

14. Blass-Kampmann S, Kindler-Rohrborn A, Deissler H, D'Urso D, Rajewsky MF. In vitro differentiation of neural progenitor cells from prenatal rat brain: common cell surface glycoprotein on three glial cell subsets. J Neurosci Res 1997;48:95-111. [CrossRef]

15. Falk $A$, Frisen J. Amphiregulin is a mitogen for adult neural stem cells. J Neurosci Res 2002;69:757-62. [CrossRef]

16. Kotani M, Osanai T, Tajima Y, Kato H, Imada M, Kaneda H, et al. Identification of neuronal cell lineage-specific molecules in the neuronal differentiation of P19 EC cells and mouse central nervous system. J Neurosci Res 2002;67:595-606. [CrossRef]

17. Li Y, Chen J, Chopp M. Cell proliferation and differentiation from ependymal, subependymal and choroid plexus cells in response to stroke in rats. J Neurol Sci 2002;193:137-46. [CrossRef]
18. Chouaf-Lakhdar L, Fevre-Montange M, Brisson C, Strazielle N, Gamra H, Didier-Bazes M. Proliferative activity and nestin expression in periventricular cells of the adult rat brain. Neuroreport 2003;14:633-6. [CrossRef]

19. Irvin DK, Nakano I, Paucer A, Kornblum HI. Patterns of Jagged 1, Jagged 2, Delta-like 1 and Delta-like 3 expression during late embryonic and postnatal brain development suggest multiple functional roles in progenitors and differentiated cells. J Neurosci Res 2004;75:330-43. [CrossRef]

20. Lendahl U, Zimmerman LB, McKay RD. CNS stem cells express a new class of intermediate filament protein. Cell 1990;60:585-95. [CrossRef]

21. Emerich DF, Schneider P, Bintz BE, Hudak J, Thanos CG. Aging reduces the neuroprotective capacity, VEGF secretion, and metabolic activity of choroid plexus epithelial cells. Cell Transplant 2007; 16:697-705. 